Portland State University

PDXScholar

Electrical and Computer Engineering Faculty

Publications and Presentations

$5-1-1983$

\title{
Radio-frequency-excited carbon dioxide metal waveguide laser
}

Jonathan Gary Grossman

Lee W. Casperson

Portland State University

O. M. Stafsudd

Follow this and additional works at: https://pdxscholar.library.pdx.edu/ece_fac

Part of the Electrical and Computer Engineering Commons

Let us know how access to this document benefits you.

\section{Citation Details}

Jonathan G. Grossman, Lee W. Casperson, Oscar M. Stafsudd. Radio-frequency-excited carbon dioxide metal waveguide laser. Applied Optics, Volume 22, Number 9 (May 1983), pp. 1298-1305.

This Article is brought to you for free and open access. It has been accepted for inclusion in Electrical and Computer Engineering Faculty Publications and Presentations by an authorized administrator of PDXScholar. Please contact us if we can make this document more accessible: pdxscholar@pdx.edu. 


\title{
Radio-frequency-excited carbon dioxide metal waveguide laser
}

\author{
Jonathan G. Grossman, Lee W. Casperson, and Oscar M. Stafsudd
}

\begin{abstract}
A new type of waveguide laser is described in which a single-surface concave metal waveguide also doubles as the grounded electrode in a radio-frequency-excited gaseous discharge. The laser's output modes and spectrum have been studied theoretically and experimentally for $\mathrm{cw} \mathrm{CO}_{2}$ operation of the laser. Overall operating efficiency and mode stability are comparable with those of conventional dielectric waveguide $\mathrm{CO}_{2}$ lasers, and advantages of the metal waveguide laser include convection cooling, intracavity mode control, and partial self-alignment.
\end{abstract}

\section{Introduction}

The majority of laser oscillators are basically Fabry-Perot interferometers containing an active medium. For many applications, this design and its variants have the advantages of simplicity and good mode control. The first ruby laser, for example, was of this type. ${ }^{1}$ On the other hand, many more exotic types of laser resonator have also been developed. In this paper we report the results of a detailed study of a waveguide laser in which the cavity modes are basically the whispering-gallery modes of a curved concave metal-strip waveguide.

The first whispering-gallery laser was a sphere of $\mathrm{CaF}_{2}: \mathrm{Sm}^{++}$in which the oscillating modes were confined by nearly total internal reflection at the interface between the laser crystal and the surrounding liquid hydrogen. ${ }^{2}$ For reasons of potentially simpler fabrication, pumping, and radiation outcoupling, much more attention has been directed toward development of lasers that are basically cylindrical in shape. An early cylindrical laser consisted of a GaAs junction laser which had been polished into the form of a cylinder 18 mils in diameter. ${ }^{3}$ In this device, the radiation was believed to propagate primarily in the radial direction within the laser, and the output would consist of a $360^{\circ}$ diskshaped radiation pattern. Similar radiation patterns have also been obtained with electron-beam-pumped

When this work was done both authors were with University of California, School of Engineering \& Applied Science, Los Angeles, California 90024. J. G. Grossman is now with Hughes Aircraft Company, Electro-Optical and Data Systems Group, El Segundo, California 90245.

Received 21 September 1982.

0003-6935/83/091298-08\$01.00/0.

(C) 1983 Optical Society of America.
CdS crystals ${ }^{4}$ and with optically pumped cylinders of Rh6G dye. ${ }^{5}$ Whispering laser modes have been observed in ruby toroids, ${ }^{6-8}$ and whispering-gallery injection lasers have been fabricated.9,10 Such whispering modes are also one of the dominant parasitic phenomena in very high-gain laser systems. ${ }^{11}$

In related developments, it has been noted that more useful electromagnetic modes might be obtainable if the outer reflecting surface were curved slightly in the direction of the axis of symmetry to reduce diffraction losses in that direction. A sketch of such a resonator is given in Fig. 1. Resonators of this type have been studied theoretically, and it is found that the field distribution perpendicular to the laser plane is expressible in terms of Hermite-Gaussian functions, the field in the radial direction involves Bessel functions, and the field in the azimuthal direction involves trigonometric functions. ${ }^{12-15}$ The low-order Bessel-function modes propagate primarily in the radial direction within the resonator, while the high-order modes are essentially whispering-gallery modes propagating along the mirror surface. $\mathrm{A} \mathrm{CO}_{2}$ laser of this type has been operated, and a radial aperture has been introduced to discriminate against the whispering-gallery modes. ${ }^{16}$

Independent of these developments concerning cylindrical laser resonators came the realization that a curved metal strip might have some important advantages for the waveguiding of electromagnetic radiation. While there has been steady progress in the development of low-loss optical fibers, there remain important spectral regions where transparent materials are not yet available. In these infrared and ultraviolet portions of the spectrum, metal waveguides are still effective, and such waveguides are also tolerant of very high power levels. The propagation of low-order modes in metal waveguides has been well-understood for many years, and curved and twisted guides have also been consid- 


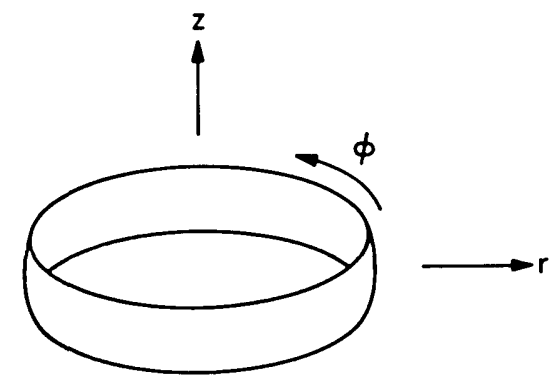

Fig. 1. Low-loss cylindrical laser resonator.

ered. ${ }^{17}$ More recently, flexible metal waveguides of rectangular cross section have been developed for transmitting infrared wavelengths, ${ }^{18-20}$ and it has been shown that effective light guiding is possible using a single curved metallic surface rather than a fully enclosed waveguide. ${ }^{21,22}$ This concept has been extended by making the curved waveguide concave and thus eliminating diffraction losses entirely. ${ }^{23-26}$ In the simplest cases, these waveguide surfaces are identical to the circularly curved mirror surface indicated in Fig. 1 , and the Airy-Hermite-Gaussian modes are special cases of the Bessel-Hermite-Gaussian modes of the radial-mode laser resonators.

The whispering-gallery waveguides can also be incorporated in Fabry-Perot-like gas laser resonators. Thus, it has been found that a cw $\mathrm{CO}_{2}$ laser with a slightly bent glass discharge tube supports modes which agree well with the theoretical Airy-Hermite-Gaussian functions. ${ }^{27}$ We report here the results of various experimental studies of a new rf-excited metal-waveguide laser oscillator. The resonator waveguide in these experiments is a concave metal strip of the same type used in earlier waveguide studies, and this waveguide surface also serves as an electrode for the rf gas discharge. As will be shown in later sections of this paper, the metalwaveguide $\mathrm{CO}_{2}$ laser has some potential advantages over conventional $\mathrm{CO}_{2}$ waveguide lasers.

There is substantial literature about rf-excited $\mathrm{CO}_{2}$ lasers. Many of the research efforts in this area involve lasers that use magnetic coupling of the rf power to the laser. While this is practical for dielectric waveguide lasers, it is not feasible for metal-waveguide lasers. The coupling of the rf power to the laser by impedance matching, however, is a practical approach, and it has been shown that the laser head can be accurately represented by an electronic model. ${ }^{28}$ Standard rfmatching techniques are therefore applicable in this approach. Using Smith chart impedance-matching techniques, it has been possible to obtain near perfect rf matching of the laser to the rf source (zero rf power reflected from the laser head), with rf coupling efficiencies as high as $85 \%-95 \%{ }^{29}$ Resistance in the network has been the main source of the rf coupling loss, with resistance as low as a tenth of an ohm leading to $10 \%$ loss. $^{30}$ Other experimental studies have included the confinement, stability, and efficiency of the rf discharge in a transverse flow $\mathrm{CO}_{2}$ laser. ${ }^{31}$
A brief summary of the mode characteristics of the concave metal surface waveguide laser is presented in Sec. II, and special attention is given to the spectral characteristics of the waveguide resonator. The $\mathrm{rf}$ properties of the laser are discussed in Sec. III, and our experimental results are presented in Secs. IV-VI.

\section{Theory}

The propagating modes of concave metal-strip waveguides have been the subject of detailed theoretical investigations. ${ }^{23,25,26}$ Only the results that are directly relevant to the present investigation will be summarized here. The coordinate system to be used in this discussion is shown in Fig. 2. The major and minor radii of curvature are $r_{0}$ and $R_{0}$, respectively, $z$ indicates distance across the waveguide surface, $r^{\prime}$ is distance perpendicular to the surface, and $\phi$ measures angular distance along the waveguide. In this system of units the main Gaussian factor in the Airy-Hermite-Gaussian mode solutions can be written ${ }^{25}$

$$
G(\phi, z)=G_{0} \exp \left\{-i\left[Q(\phi) z^{2} / 2+S(\phi) z\right]\right\},
$$

where the $Q$ and $S$ parameters are governed by the equations

$$
\begin{aligned}
Q^{2}+\frac{k_{0}}{r_{0}} \frac{d Q}{d \phi}+\frac{k_{0}^{2}}{r_{0} R_{0}} & =0, \\
Q S+\frac{k_{0}}{r_{0}} \frac{d S}{d \phi} & =0 .
\end{aligned}
$$

In these equations $Q$ is the usual beam parameter

$$
Q(\phi)=\frac{k_{0}}{R(\phi)}-i \frac{2}{w^{2}(\phi)},
$$

where $k_{0}$ is essentially the free-space propagation constant, and $R$ and $w$ are the radius of curvature of the phase fronts and the 1/e amplitude spot size in the $z$ direction, respectively. The ratio $d_{a}=-S_{i} / Q_{i}$ is the displacement in the $z$ direction of the amplitude center of the beam, and the ratio $d_{p}=-S_{r} / Q_{r}$ is the displacement in the $z$ direction of the phase center of the beam. The subscripts $i$ and $r$ denote the imaginary and real parts of the parameters $Q$ and $S$, respectively.

Equations (2)-(4) can be solved analytically to obtain the $\phi$ dependence of the beam displacement, spot size, and phase-front curvature for an arbitrary input beam. However, only the matched-mode solutions are needed

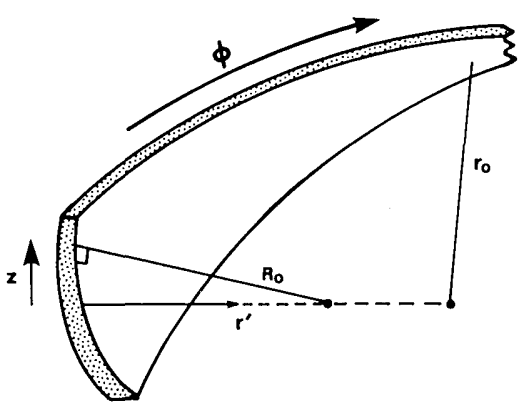

Fig. 2. Coordinate system used in the analysis of a metal waveguide laser. 


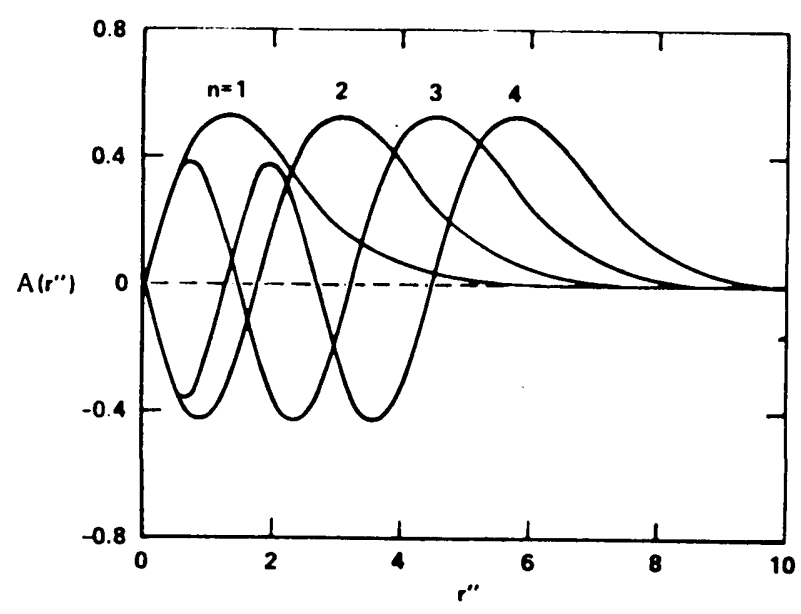

Fig. 3. Low-order Airy function radial modes of a metal strip waveguide.

in the present study. With a perfectly matched input, the steady-state propagating beam has flat phase fronts $R=\infty$ (in the $z$ direction), and the spot size is

$$
w=\left[2\left(r_{0} R_{0}\right)^{1 / 2} / k_{0}\right]^{1 / 2} .
$$

If the matched beam is misaligned with respect to the waveguide axis, the beam displacement oscillates according to

$$
\begin{aligned}
d_{a}\left(r_{0} \phi\right)= & d_{a}(0) \cos \left[\left(r_{0} / R_{0}\right)^{1 / 2} \phi\right]+d_{a}^{\prime}(0)\left(r_{0} R_{0}\right)^{1 / 2} \\
& \times \sin \left[\left(r_{0} / R_{0}\right)^{1 / 2} \phi\right],
\end{aligned}
$$

where $d_{a}(0)$ is the displacement at the input to the waveguide, and $d_{a}^{\prime}(0)$ is the input slope with respect to the $z=0$ surface. This displacement formula is useful if the resonator mirrors at the ends of the waveguide are misaligned.

The Hermite factor $H(\zeta)$ is a solution of the Hermite differential equation

$$
\frac{d^{2} H}{d \zeta^{2}}-2 \zeta \frac{d H}{d \zeta}+2 m H=0
$$

where $\zeta$ is the displaced $z$ coordinate

$$
\zeta=\left(-Q_{i}\right)^{1 / 2}\left(z-d_{a}\right)
$$

and $m$ is an integer denoting the transverse-mode order in the $z$ direction.

The Airy factor $A(\rho)$ is a solution of the Airy differential equation

$$
\frac{d^{2} A}{d \rho^{2}}-\rho A=0
$$

where $\rho$ is the displaced radial coordinate

$$
\rho=\left(2 k_{0}^{2} / r_{0}\right)^{-2 / 3}\left[\left(2 k_{0}^{2} / r_{0}\right) r^{\prime}-\alpha\right],
$$

and the constant $\alpha$ must be chosen in such a way that a zero of the Airy function occurs at the waveguide surface $r^{\prime}=0$.

Finally, the Airy-Hermite-Gaussian modes must also include an exponential phase factor of the form

$$
F(\phi)=F_{0} \exp [-i P(\phi)],
$$

where the phase $P(\phi)$ is governed by the equation

$$
\frac{1}{r_{0}} \frac{d P}{d \phi}=-\frac{i Q_{r}}{2 k_{0}}+\frac{(m+1 / 2) Q_{i}}{k_{0}}-\frac{S^{2}+\alpha}{2 k_{0}} .
$$

When these various factors are multiplied together, one obtains a complete set of eigenmodes for the concave metal-strip waveguide. Most aspects of these modes are well-known. For reference, several of the low-order Airy function solutions of Eq. (9) are plotted in Fig. 3 as functions of the coordinate $r^{\prime \prime}$, which measures distance away from the waveguide surface in units of $\rho$. It is evident from this figure that, with increasing mode order, the field extends farther away from the waveguide surface. In an rf-excited metal waveguide laser, therefore, the gap between the electrodes may limit the number of possible resonator modes.

The output spectrum of the laser may be found by requiring that the total round-trip phase delay be an integer multiple of $2 \pi$. For the most important nonpinching steady-state modes $\left(Q_{r}=S=0\right)$, Eq. (12) reduces to

$$
\frac{1}{r} \frac{d P}{d \phi}=-\frac{m+1 / 2}{\left(r_{0} R_{0}\right)^{1 / 2}}-\frac{\alpha}{2 k_{0}},
$$

where Eqs. (4) and (5) have been used to evaluate $Q_{i}$. This equation can now be integrated together with the ordinary plane-wave phase exponential to yield the oscillation condition

$$
2 \pi q=2 r_{0} \phi_{0} \frac{m+1 / 2}{\left(r_{0} R_{0}\right)^{1 / 2}}+2 r_{0} \phi_{0} \frac{\alpha_{n}}{2 k_{0}}+2 k_{0} r_{0} \phi_{0},
$$

where $q$ is an integer, $\alpha_{n}$ denotes the $\alpha$ value corresponding to the $n$ th-order Airy function modes, and $\phi_{0}$ is the angular length of the cavity. It follows from Eq. (14) that the cavity mode spacings are

$$
\begin{aligned}
\Delta \nu_{\text {longitudinal }} & =\frac{c}{2 r_{0} \phi_{0}}, \\
\Delta \nu_{\text {transverse-Hermite }} & =\frac{c}{2 \pi\left(r_{0} R_{0}\right)^{1 / 2}}, \\
\Delta \nu_{\text {transverse-Airy }} & =\frac{c\left(\alpha_{n}-\alpha_{n-1}\right)}{4 \pi k_{0}} .
\end{aligned}
$$

These results are compared with experimental data in a later section of this paper.

\section{III. rf Properties}

To design a metal-waveguide laser having reasonable efficiency in converting rf power to laser power, the rf properties of the laser must be understood. The purpose of this section is to develop simple analytical models which can be used as guidelines in designing a metal-waveguide rf laser discharge. As a first step, some approximate constraints on the rf frequency will be established.

It has been shown that, in the presence of an rf field, a plasma has a current density governed by 29

$$
J(t)=\frac{N_{e} e^{2} E \cos (\omega t-\phi)}{m_{e}\left(\nu^{2}+\omega^{2}\right)^{1 / 2}},
$$

where $N_{e}$ is the electron density, $e$ is the electron charge, $E$ is the electric-field amplitude, $m_{e}$ is the electron mass, $\nu$ is the collison frequency of electrons with gas molecules, $\omega$ is the angular frequency of the applied rf field, 
and $\phi$ is a phase lag $(\tan \phi=\omega / \nu)$. This result is based on several assumptions including: (1) ion motion is neglected since the ions are much more massive than the electrons; (2) the electron molecule collision frequency is much greater than the $\mathrm{rf}$ frequency $\left(\nu \sim 2 \times 10^{11}\right.$ $\left.\mathrm{sec}^{-1}\right)$; (3) induced moments do not greatly perturb the vacuum values of $\mu$ and $\epsilon$; and (4) rf magnetic effects are neglected because the electron drift velocities are small.

Equation (18) may be used to derive a constraint on the rf frequency. This frequency must be high enough that the majority of electrons in the discharge are prevented from hitting the electrodes. More specifically, the average drift range $x(t)$ must be much less than the electrode spacing. The drift range can be found by using $J(t)=N_{e} e d x / d t$ and integrating Eq. (18). The result is

$$
x(t)=\frac{e E \sin (\omega t-\phi)}{m_{e} \omega\left(\nu^{2}+\omega^{2}\right)^{1 / 2}} .
$$

If the electrode spacing is $d$, the condition $2 x_{\max } \ll d$ may be written as a constraint on the frequency

$$
f \gg \frac{e V}{\pi m_{e} d^{2} \nu}=11 \mathrm{MHz},
$$

where $\nu \gg \omega$, and a voltage $V=E d=100 \mathrm{~V}$ was assumed together with an electrode spacing of $1.5 \mathrm{~mm}$.

An upper limit on the rf frequency can be obtained from the requirement that the discharge be spatially uniform. This requirement is most easily met if the discharge length is kept to a small fraction of the rf wavelength to prevent standing-wave patterns from developing along the electrode structure. For example, if the rf wavelength is at least four times the discharge length, one obtains the approximate condition

$$
f<\frac{c}{4 l}=375 \mathrm{MHz},
$$

where a reasonable discharge length of $l=0.2 \mathrm{~m}$ has been assumed. Thus, for a typical waveguide laser the possible values of the drive frequency are bounded by $11 \mathrm{MHz} \ll f<375 \mathrm{MHz}$.

It is also helpful to develop a circuit model of the laser. The laser head can be imagined as a pair of parallel plates separated by a gap filled with the rf discharge. Ignoring the distributive effects of the plates, the circuit model of the laser is basically a parallel RC circuit, with its resistance that of the discharge and its capacitance that of the parallel plates. For completeness, stray input capacitance, resistance, and inductance should also be included to represent the parasitic impedance present in real wires, $r f$ feedthroughs, etc. The values of these stray impedances are difficult to calculate, but measured values are available for many types of rf-excited laser. ${ }^{29-31}$ Figure 4 shows the lumped circuit model used in our studies, and this model can be used to determine the response of the laser to various conditions of rf pumping.

An important application of the circuit model of the laser is in the development of an impedance-matching network. It would not normally be expected that the impedance of the laser would match the $50 \Omega$ impedance typical of many rf power supplies, so a matching network is required to achieve a reasonable level of efficiency. Several different types of matching network could be used, and these networks would differ primarily in their response to frequency shifts or other power supply transients. We have used a capacitance-matching network because of its wide frequency tunability (using adjustable capacitors). Optimum element values can be determined algebraically or by means of standard Smith chart methods, and the details are omitted here.

The overall efficiency of the laser is, in part, determined by the ratio of the volume of gas ionized by the rf fields to the volume occupied by the waveguide modes. The discharge volume is defined by the electric fields, the electronic motions, and the molecular mean free path. The ratio of the rf electric field to the molecular density of the gas must be above $5 \times 10^{-16} \mathrm{~V} \mathrm{~cm}{ }^{2}$ for ignition of the discharge to occur. ${ }^{29,30}$ Blooming of the discharge happens when the fringing fields close to the electrode gap are intense enough to sustain the discharge, and thermal motions allow it to expand into this volume. The blooming volume, however, is found to be a small $(<20 \%)$ part of the discharge volume. ${ }^{30}$

The major cause of discharge blooming is the drift velocity of the electrons. For most $\mathrm{CO}_{2}$ rf-excited lasers, the electron drift velocity is $\sim 7 \times 10^{6} \mathrm{~cm} / \mathrm{sec}^{29}$ Assuming that no unfavorable collisions occur during half an rf period, the distance traversed by the electrons is on the order of

$$
x_{0}=\frac{7 \times 10^{6}}{2 f}=0.23 \mathrm{~mm} \text { at } f=150 \mathrm{MHz} .
$$

This is $\sim 10 \%$ of the discharge width and leads to a $20 \%$ volume increase when both sides are considered. The rf discharge and, therefore, the laser's operating efficiency should show better performance at the higher $\mathrm{rf}$ drive frequencies.

\section{Laser Design and Construction}

Experimental studies have been undertaken to confirm the theoretical results of the previous sections. These studies have involved construction of two $\mathrm{CO}_{2}$ metal-waveguide lasers. The need for two different lasers is related to two different aspects of the theoretical results. Specifically, to measure the frequency properties of the laser, we require it to run on multiple transverse modes, whereas to measure the output beam

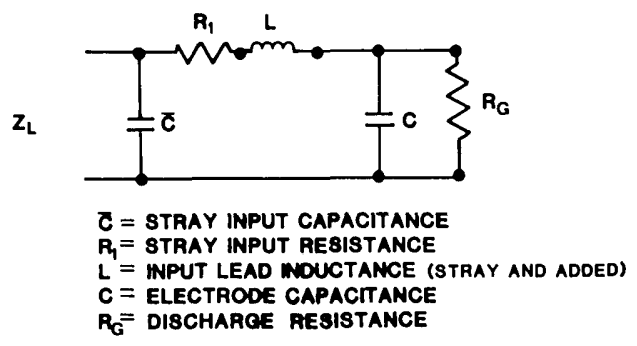

Fig. 4. Laser-head lumped circuit model. 


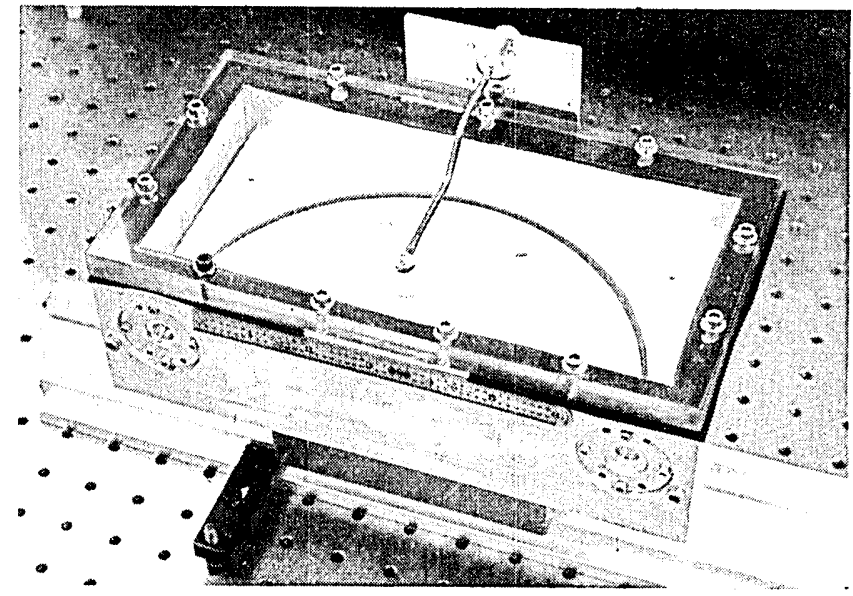

Fig. 5. Photograph of rf-excited $\mathrm{CO}_{2}$ metal waveguide laser.

intensity profile, a single transverse mode is necessary. A laser designed to run on higher-order transverse modes must have, as in the metal waveguide laser, losses that are nearly independent of mode order. ${ }^{22}$ On the other hand, a single-mode laser must have losses that increase rapidly with mode order.

The design of a multimode metal-waveguide laser requires the gap between the rf electrodes to be several times greater than the lowest transverse-order mode thickness. If we define the Airy mode thickness as the distance between the waveguide and the $e^{-2}$ beam intensity point, this thickness is given approximately from Eqs. (9) and (10) by

$$
\Delta r=3\left(r_{0} / 2 k_{0}^{2}\right)^{1 / 3} .
$$

Therefore, to determine the longitudinal radius for a multimode metal-waveguide laser the electrode gap must be known. Most rf-excited $\mathrm{CO}_{2}$ lasers have an electrode gap of 1-4 $\mathrm{mm}$. This is due to the difficulty of striking an rf discharge for electrode gaps $>4 \mathrm{~mm}$ and the high probability of discharge arcs occurring for gaps $<1 \mathrm{~mm}$

Starting from considerations like the foregoing, it has been concluded that a $0.1-\mathrm{m}$ radius spherical waveguide laser with a 3-mm electrode gap would exhibit both multiple-order transverse Airy modes and multiple Hermite-Gaussian transverse modes. Our experimental multimode laser was based on a spherical waveguide of this radius in which the $180^{\circ}$ waveguide length is terminated by flat mirrors. A photograph of the device is given in Fig. 5. Larger radii would have resulted in the laser running only on the lowest-order transverse-Gaussian mode, while smaller radii would have required too small a discharge length. Our single-mode laser has a 40-m longitudinal radius, a $6-\mathrm{mm}$ transverse radius, a $3-\mathrm{mm}$ electrode gap, and a cavity length of $0.23 \mathrm{~m}$.

The optics used for both lasers include flat $95 \%$ reflecting $\mathrm{ZnSe}$ output couplers and flat highly reflecting dielectric-coated germanium back reflectors. Alignment of the lasers is performed by retroreflecting a $6328-\AA \mathrm{He}-\mathrm{Ne}$ beam. The lasers are normally operated with flowing gas and convection cooling. A 3:1:1 mixture of $\mathrm{He}, \mathrm{CO}_{2}$, and $\mathrm{N}_{2}$ is used in the lasers, which as shown in Fig. 6 produces maximum laser output power at 55-torr total laser cavity pressure. Short-term sealed-off operation of the lasers is also optimized at a pressure of 55 torr.

The coupling of the rf power to the laser is performed in the manner shown in Fig. 7. The rf signal from a master oscillator is amplified and delivered to a capacitive $\pi$ matching network through a rf power meter. Typically, between $20-100 \mathrm{~W}$ of rf power are required to turn on the laser depending on the cavity pressure. The matching network consists of two $5-25 \mathrm{pF}$ variable piston-type capacitors, with one capacitor in series with the laser head and the other in parallel.

The main concern about the rf drive frequency is its effects on the laser's output power and modes. The laser's output power is proportional to the fraction of the rf power delivered to the volume defined by the laser modes. For the case of the multimode laser, this volume is related to the electrode gap. As the discharge blooms beyond this volume, the laser output power should decrease by the ratio of the discharge blooming volume to the original discharge volume. In Sec. III, the discharge was calculated to expand under worst-case assumptions by $\sim 20 \%$. Therefore, a similar variation in the laser's output power should be seen as the rf drive frequency is varied, with the higher output powers occurring at the higher frequencies.

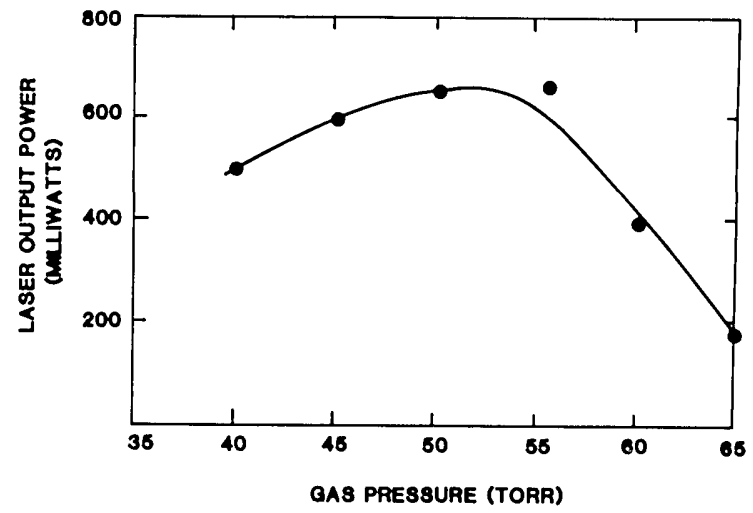

Fig. 6. Laser output power vs gas pressure.

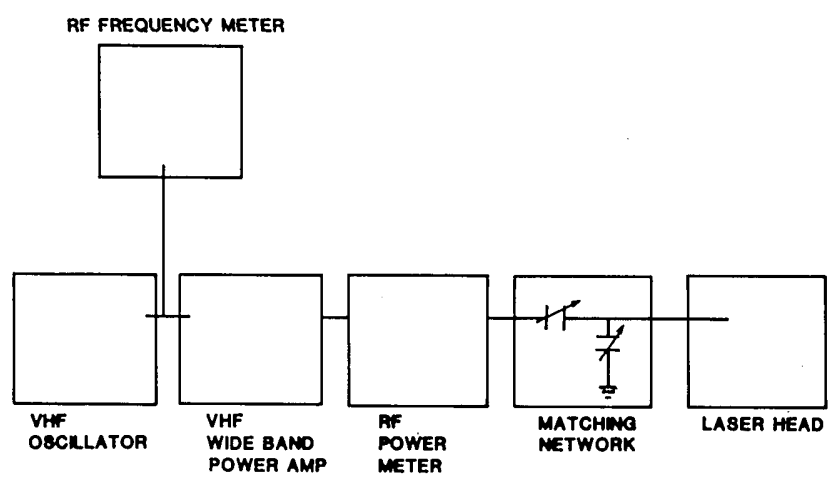

Fig. 7. Rf power electronics arrangement. 


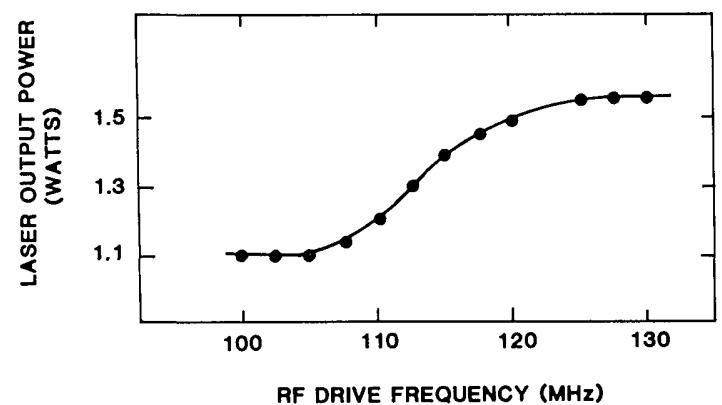

Fig. 8. Laser output power vs rf drive frequency at $50-\mathrm{W}$ rf power, 50 -torr pressure, and a gas mixture of $3: 1: 1, \mathrm{He}: \mathrm{CO}_{2} \mathrm{~N}_{2}$.

Table I. Multimode Laser's Calculated Mode Spacings

\begin{tabular}{lc}
\hline \multicolumn{1}{c}{ Mode } & Frequency spacing \\
\hline Longitudinal & $480 \mathrm{MHz}$ \\
Transverse Hermite-Gaussian & $477.6 \mathrm{MHz}$ \\
Transverse Airy & $14.89 \mathrm{GHz}$ \\
\hline
\end{tabular}

To help confirm that it is the discharge blooming that is causing the variations in the laser output power, the laser's output modes and wavelengths must be shown to be constant as the rf drive frequency is changed. This was accomplished by splitting the laser beam into two parts. A portion of the beam is viewed on a thermal image plate and examined for changes in its mode structure. The remainder of the laser beam enters an Optical Engineering $\mathrm{CO}_{2}$ spectrum analyzer, and its wavelength structure is measured. The rf drive frequency is varied from 100 to $135 \mathrm{MHz}$, while holding the rf power level at $50 \mathrm{~W}$. No changes in the laser's mode pattern or wavelength $(10.50 \mu \mathrm{m})$ were observed. Visual inspection of the discharge, however, shows it to bloom more at the lower drive frequencies than at the higher frequencies.

The changes in the laser's output power as the drive frequency is varied were monitored by a Scientech model 362 power meter placed directly in front of the laser. Figure 8 shows the results. The laser output power is seen to vary by $\sim 30 \%$ over the $100-135-\mathrm{MHz}$ rf drive frequency range measured. This result closely matches the theoretical expectations, which suggests that the assumptions made in Sec. III are valid for this laser.

\section{Output Spectrum Experiments}

The frequency difference between the modes of a metal waveguide laser are, as derived in Sec. II, dependent only on the waveguide electrode geometry and the optical length of the laser. The actual output spectrum of the laser, however, is also limited by the linewidth of the $\mathrm{CO}_{2}$ transition and the mode selectivity of the laser. The design of our multimode laser enables it to operate on transverse modes as high as the second-order Hermite-Gaussian, ninth-order Airy mode. Since the laser is not selective between lower-order transverse waveguide modes, the output will be multimode. The mode frequency spacings for the multimode laser are calculated from Eqs. (15)-(17) and are listed in Table I.

The laser's longitudinal and transverse-mode spacings are designed to be slightly different to enable straightforward interpretation of the measurements of the laser's output spectrum. This unfortunately leads to an Airy mode spacing far greater than the other mode spacings. To compound this problem, the transition linewidth for a $\mathrm{CO}_{2}$ laser operating at 50 torr is only 300 $\mathrm{MHz}^{32}$ This implies that the laser will only operate on multiple transverse Airy-function modes if each transverse mode has a different longitudinal-mode number associated with it as shown in Fig. 9.

Figure 10 shows the experimental arrangement used to verify the previous conclusions. The multimode laser's output is directed through a II-VI, Inc. model PAZ-15-AC variable attenuator/polarizer to the S.A.T. model A4 HgCdTe photodiode. The photodiode is positioned to intercept all the transverse modes of the laser. The output of the detector is amplified and then directed to a Tektronix model 7L13 spectrum analyzer which displays the diode's rf spectrum on a Tektronix model 7400 storage oscilloscope. The variable attenuator/polarizer is used to control the amount of laser power incident on the detector and to determine the polarization of the laser output. The $\mathrm{HgCdTe}$ photodiode has a bandwidth of $75 \mathrm{MHz}$ when cooled to $77 \mathrm{~K}$ and reverse biased to $-0.5 \mathrm{~V}$. The bias is provided by the amplifier, which also provides the $50-\Omega$ impedance load required for the photodiode and a voltage gain of 100.

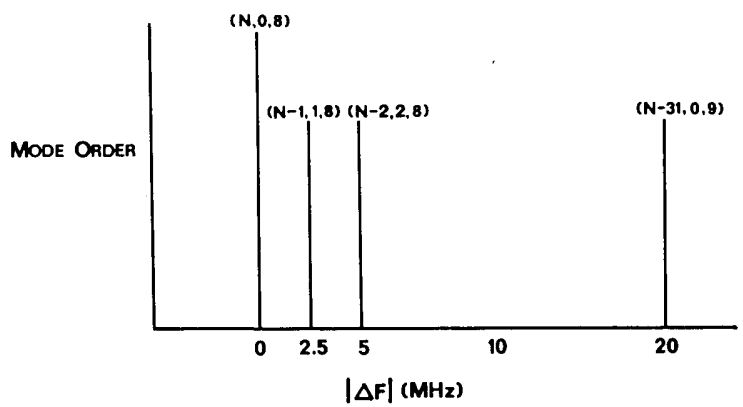

Fig. 9. Relative mode spacings for the multimode laser. The numbers in parentheses correspond to longitudinal mode number, transverse Hermite-Gaussian mode number, and transverse Airymode number, respectively.

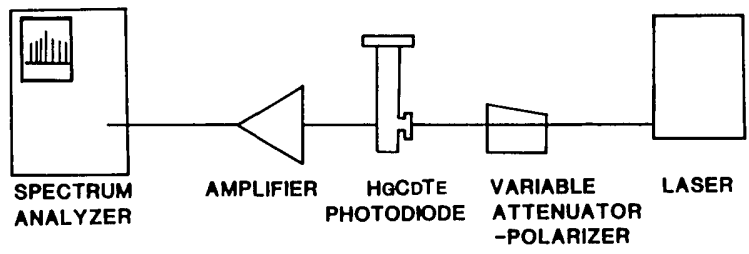

Fig. 10. Experimental arrangement for measuring the laser output spectrum. 
Figure 11 shows the output spectrum of the laser. The 2.4-MHz signal matches the value calculated for the transverse Hermite-Gaussian mode spacings. To prove this is the case, an aperture was placed intracavity to suppress the higher-order Hermite-Gaussian modes. With the aperture in place, the laser output was observed to be lowest-order Hermite-Gaussian with the output spectrum shown in Fig. 11(b). Only the 15- MHz transverse Airy-mode spacing is observed in this figure. The polarization of the laser should be parallel to the waveguide surface as this represents the lowest-loss

$\mathrm{OHz}$

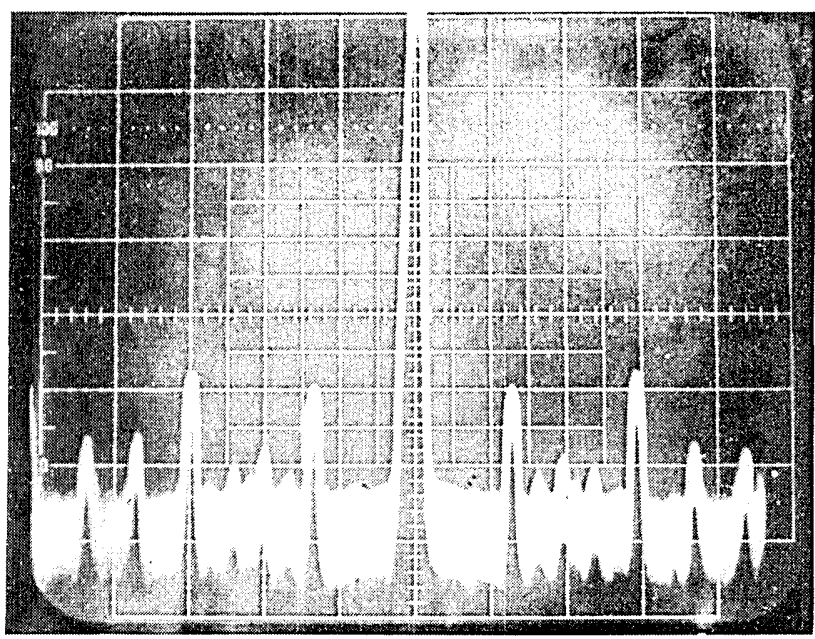

(a)

\section{$\mathrm{OHz}$}

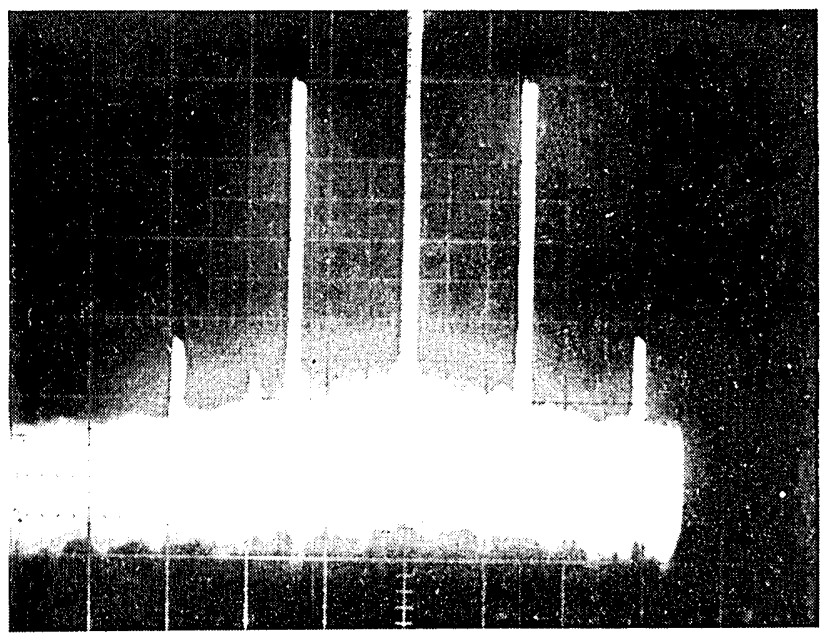

(b)

Fig. 11. Laser output spectrum for (a) no intracavity aperture, 2$\mathrm{MHz} /$ div horizontal, arbitrary units vertical, and (b) intracavity aperture, $10-\mathrm{MHz} / \mathrm{div}$ horizontal, arbitrary units vertical. waveguide polarization. This conclusion has been experimentally verified using the polarizer indicated in Fig. 10.

\section{Waveguide Mode Experiments}

The mode properties of the concave metal waveguides have been examined in Sec. II, and two important conclusions have been derived. The first is that the field profile of the waveguide modes is represented by Airy functions perpendicular to the waveguide surface and Hermite-Gaussian functions parallel to the waveguide. The second is the existence of stable off-center waveguide modes. Unlike the modes of other waveguides, the concave metal waveguide modes can oscillate transverse to the waveguide as they propagate along it, and this effect has been shown to be useful for the case of the misaligned waveguide laser. As discussed below, these results have been verified experimentally using our low-order-mode waveguide laser.

The intensity profile of the laser has been measured with a rotating mirror and a small area $\left(0.04-\mathrm{cm}^{2}\right)$ photovoltaic $\mathrm{HgCdTe}$ detector. With the detector in the near field of the laser modes, various mode scans were made. Rotating the mirror about the $z$ axis of the waveguide, the laser is found to operate lowest-order transverse mode only when the laser is properly aligned. It is found that, under severe misalignment of the laser (both optics and top rf electrode tilted $\sim 2^{\circ}$ each), the laser output is a second-order transverse Airy mode. In normal operating conditions, however, the second-order mode is not observed. In both cases the observed modes are well-represented by slightly diffracted Airy function modes. Scans along the Gaussian axis of the waveguide have also been made and found to be within $\sim 1 \%$ of the calculated beam profile.

The propagation of an off-center mode is given by Eq. (6). In the case of the metal-waveguide laser, $d_{a}^{\prime}(0)$ represents the tilt of the beam to the waveguide axis at the plane $r \phi=0$, and $d_{a}(0)$ can take on any value that enables the waveguide mode to be within the gain region of the laser. Thus, the oscillatory nature of the solutions in Eq. (6) suggests that, for certain misalignments of the laser's mirrors, stable resonator modes exist. Numerical evaluations of Eq. (6) for the lasers built for this study show that, for small values of $d_{a}^{\prime}(0)$, stable resonator modes are possible. By rotating the rear reflector of each laser around the $r$ axis of the waveguide, it has been found that rotations as large as $0.5^{\circ}$ do not affect the laser's output power. The polarization of the output, however, changes its direction to maintain itself parallel to the waveguide surface.

\section{Discussion}

A new type of waveguide laser has been described. The waveguide is a single concave metal surface that also doubles as the grounded electrode in a radio-frequency-excited gaseous discharge. Theoretical studies on the modes and frequency characteristics of the laser have been performed, and two metal-waveguide carbon dioxide lasers have been built to verify the theoretical results. One laser is designed to run multimode, and 
the other is designed to run lowest-order transverse mode. Both lasers demonstrate output powers in the 1-2-W range, with operating efficiencies comparable to those of conventional $\mathrm{CO}_{2}$ lasers. Practical advantages of these lasers in comparison with other waveguide lasers include reduced fabrication costs, ruggedness, improved mode control, and ultimately perhaps higher operating efficiency.

The standard rf waveguide lasers have a rectangular cross section in which two of the sides are metallic rf electrodes and the remaining two sides are a dielectric material (usually ceramic), the principal function of which is to confine the laser radiation into a rectangular waveguide mode. Although this design has many desirable features, as proved by its commercial success, the need for dielectric waveguide walls is a remaining undesirable feature. Suitable dielectric components are costly and difficult to fabricate, and they also may limit laser performance and lifetime. In the new laser designs described here, the metal electrodes are curved slightly to ensure beam confinement, and the costly and sometimes toxic ceramic sides of the laser waveguide are dispensed with altogether. Elimination of these delicate ceramics also leads to increased ruggedness and may reduce the impurity levels, which limit the laser lifetime.

Other advantages of the new waveguide laser design may result from the possibility of transverse gas flow. Natural or forced convection can be employed to remove heat from the discharge region, and overheating is one of the most important factors limiting power, efficiency, and lifetime in conventional waveguide lasers. In addition, the open sides of the waveguide eliminate the large pressure waves normally associated with pulsed operation. Transverse mode control is another major difficulty in the design and operation of conventional waveguide lasers. With the new design described here, parameters that govern the laser mode structure are independent from the parameters that govern the gas discharge conditions. Besides this added design flexibility, the natural modes of a curved waveguide already have the very desirable Gaussian beam form in one transverse direction and may be transformed to Gaussian beam form in the other direction. In waveguide lasers, because of their small size and large mode spacings, mode control is important for both its spatial and spectral consequences.
The authors are pleased to acknowledge useful discussions of this research with F. G. Allen, D. Fink, M. F. Nicol, R. A. Satten, and L. V. Sutter.

\section{References}

1. T. H. Maiman, Nature (London) 187, 493 (1960).

2. C. G. B. Garrett, W. Kaiser, and W. L. Bond, Phys. Rev. 124, 1807 (1961).

3. K. M. Arnold and S. Mayburg, J. Appl. Phys. 34, 3136 (1963).

4. F. H. Nicoll, IEEE J. Quantum Electron. QE-4, 198 (1968).

5. Z. G. Horvath, A. V. Kilpio, A. A. Malyutin, and Y. N. Serkyuchenko, Opt. Commun. 35, 142 (1980).

6. D. Roess, Proc. IEEE 51, 468 (1963).

7. D. Walsh and G. Kemeny, J. Appl. Phys. 34, 956 (1963).

8. D. Roess and G. Gehrer, Proc. IEEE 52, 1359 (1964).

9. N. Matsumoto and K. Kumabe, Jpn. J. Appl. Phys. 16, 1395 (1977).

10. I. Ury, S. Margalit, N. Bar-Chaim, M. Yust, D. Wilt, and A. Yariv, Appl. Phys. Lett. 36, 629 (1980).

11. S. N. Suchard, R. L. Kerber, G. Emanuel, and J. S. Whittier, J. Chem. Phys. 57, 5065 (1972), and references therein.

12. L. A. Wainstein, High-Power Electron. 3, 176 (1964).

13. G. Goubau and F. Schwering, IEEE Trans. Microwave Theory Tech. MTT-13, 749 (1965).

14. E. A. J. Marcatili, U.S. Patent 3,241,085 (1966).

15. L. W. Casperson, J. Opt. Soc. Am. 63, 25 (1973).

16. L. W. Casperson and C. Romero, IEEE J. Quantum Electron. QE-9, 484 (1973).

17. See, for example, L. Lewin, Proc. Inst. Electr. Eng. 102B, 75 (1955).

18. E. Garmire, T. McMahon, and M. Bass, Appl. Opt. 15, 145 (1976).

19. E. Garmire, T. McMahon, and M. Bass, Appl. Phys. Lett. 29, 254 (1976).

20. E. Garmire, T. McMahon, and M. Bass, Appl. Phys. Lett. 31, 92 (1977).

21. H. Krammer, Appl. Opt. 16, 2163 (1977).

22. H. Krammer, Appl. Opt. 17, 316 (1978).

23. M. E. Marhic, L. I. Kwan, and M. Epstein, Appl. Phys. Lett. 33, 609 (1978).

24. M. E. Marhic, L. I. Kwan, and M. Epstein, Appl. Phys. Lett. 33, 874 (1978).

25. L. W. Casperson and T. S. Garfield, IEEE J. Quantum Electron. QE-15, 491 (1979).

26. M. E. Marhic, J. Opt. Soc. Am. 69, 1218 (1979).

27. M. E. Marhic, L. I. Kwan, and M. Epstein, IEEE J. Quantum Electron. QE-15, 487 (1979).

28. K. D. Laakman, U.S. Patent 4,169,251 (1979).

29. G. A. Griffith, Hughes Aircraft Co.; unpublished correspondence (1979).

30. L. V. Sutter, Hughes Aircraft Co.; unpublished correspondence (1980).

31. J. H. Wang and J. N. Parento, Hughes Aircraft Co.; unpublished correspondence (1981).

32. A. Yariv, Quantum Electronics (Wiley, New York, 1975), p. 217. 